\title{
Talokrurale Kugelgelenkbildung: Eine unauffällige Fehlbildung mit wichtigen Begleitsymptomen
}

Das normale obere Sprunggelenk ist ein Scharniergelenk mit rechteckigem Querschnitt in der Frontalebene und halbkreisförmigem Querschnitt in der Sagittalen. Lamb beschrieb als erster fünf Fälle eines oberen Sprunggelenks mit halbkreisförmiger Gelenkfläche in frontaler und sagittaler Ansicht; das distale Ende der Tibia hat dabei eine kongruente konkave Gelenkfläche (Lamb, J Bone Joint Surg 1958; 40B: 240 - 243). Seitdem ist diese Deformität in der Literatur als „Ball-and-Socket Ankle“ beschrieben. Während das Sprunggelenk selbst nur wenig Beschwerden verursacht, ist mit dieser Anomalie eine Reihe anderer Deformitäten assoziiert, die klinisch bedeutend sind.

\section{Fallbeschreibung}

Ein 15-jähriger männlicher Patient wurde zur operativen Korrektur einer Beinlängendifferenz vorgestellt. Es bestand eine Verkürzung des rechten Beines um $5,5 \mathrm{~cm}$ mit der größten Längendifferenz distal des Knies; $1 \mathrm{~cm}$ Längendifferenz bestand allein im Rückfuß. Der rechte Fuß war 4-strahlig und um 2,5 cm kürzer als der linke. Der Bewegungsumfang in Hüfte, Knie- und Sprunggelenk war seitengleich.

Der Patient war das Kind nicht verwandter gesunder Eltern. Der Verlauf der Schwangerschaft war unauffällig. Bei der Geburt bestanden außer der Oligodactylie keine Auffälligkeiten, insbesondere keine Beinlängendifferenz. Bei sonst normaler Entwicklung fiel eine zunehmende Verkürzung des rechten Beines auf, die ab dem 5. Lebensjahr durch Schuhzurichtung ausgeglichen wurde. Eine leichte Instabilität im Sprunggelenk hatte keine klinische Konsequenz.

Röntgenaufnahmen mit 15 Jahren zeigten rechtsseitig ein Ball-and-Socket Ankle (Abb.1); am Fuß fehlte der vierte Zehenstrahl (Abb.2). Läsionen als Ursache der Beinverkürzung bestanden nicht. Die Beinlängendifferenz wurde durch eine Wagnersche Distraktion ausgeglichen. Im Alter von 21 Jahren wurden wegen einer Außenbandruptur gehalte-

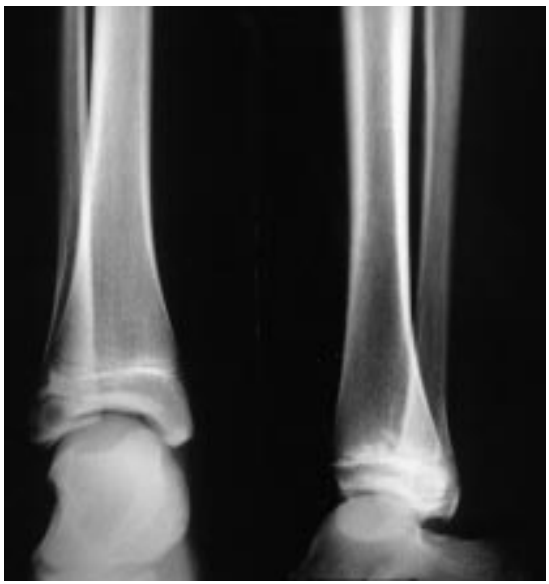

Abb.1 Rechtes Sprunggelenk im Alter von 15 Jahren a.p. und seitlich. Es bestehen eine Kugelgelenkbildung des oberen Sprunggelenks und ein hochstehender Außenknöchel. Das Bein war zu diesem Zeitpunkt nicht operiert.

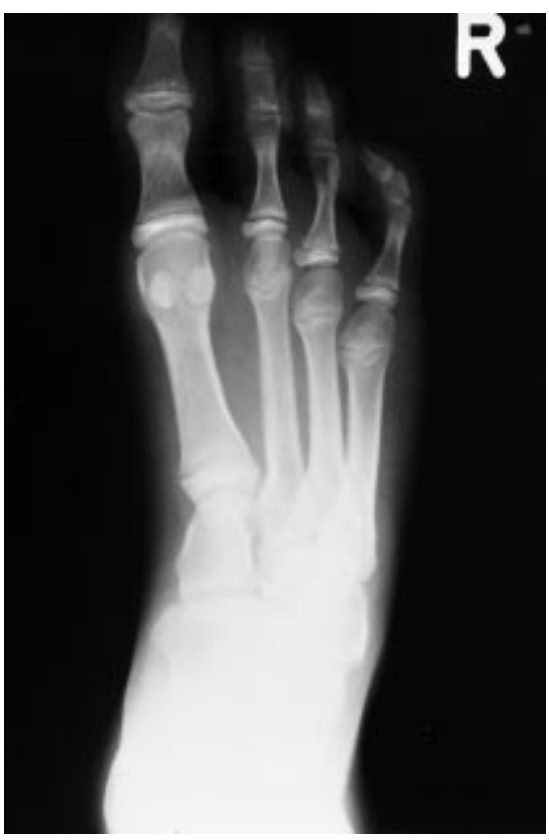

Abb. 2 Rechter Fuß mit nun vier Strahlen im Alter von 15 Jahren. Das Os cuboideum artikuliert nur mit einem statt normal zwei Zehenstrahlen, so dass ein lateraler Strahl fehlt.

ne Röntgenaufnahmen angefertigt. Sie zeigten eine normale Position des Talus bei forcierter Eversion des Fußes und 40 Grad Rotation bei forcierter Inversion 


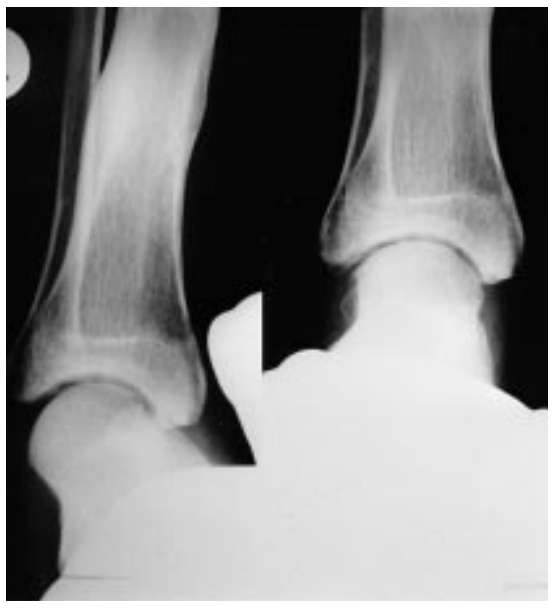

Abb.3 Gehaltene Aufnahmen in Inversion und Eversion nach Außenbandruptur im Alter von 21 Jahren. Bei forcierter Eversion steht der Talus regelrecht, bei forcierter Inversion finden sich $40^{\circ}$ Rotation.

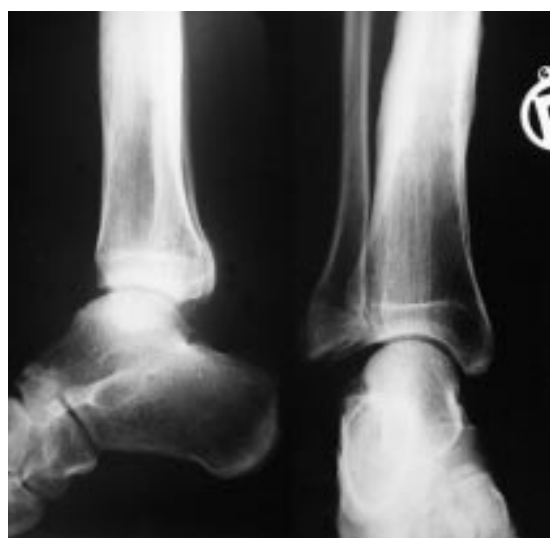

Abb.4 Rechtes Sprunggelenk seitlich und a.p. im Alter von 21 Jahren. Zusätzlich zur Kugelgelenkbildung besteht eine knöcherne Fusion von Talus und Kalkaneus.

(Abb. 3). In einer seitlichen Aufnahme erkennt man eine Fusion von Talus und Kalkaneus (Abb. 4). Die Bandruptur wurde operativ versorgt; weitere Ereignisse traten nicht auf.

\section{Diskussion}

Der Patient zeigte einen für das Ball-andSocket Ankle typischen Verlauf. In der Literatur wurden bisher 31 Arbeiten mit insgesamt 273 Fällen des Ball-and-Socket Ankle bei 235 Patienten gefunden. Die Deformität ist knabenwendig $(\mathrm{m}: \mathrm{w}=2: 1)$ und tritt überwiegend einseitig, meist rechtsseitig auf. Das mittlere Alter bei Diagnosestellung liegt bei 9,6 Jahren ( 2 bis 50 Jahre). Der häufigste Vorstellungsgrund ist eine Beinlängendifferenz (86\%); Fußdeformi- täten wie Klumpfuß, Metatarsus adductus varus und Hohlfuß (zusammen 7\%) und Beschwerden wegen des Sprunggelenks (4,5\%) sind dagegen nur selten Gründe für den Arztbesuch. Der Bewegungsumfang in Inversions-/Eversionsrichtung und in Dorsal-/Plantarflexion wird für den betroffenen Fuß als gleich, verringert oder vergrößert angegeben.

Eine Beinverkürzung ist auch das am häufigsten assoziierte Symptom (90\%), mit im Mittel $5 \mathrm{~cm}$ Längendifferenz, die am Unterschenkel überwiegt. Tarsale Synostosen sind am zweithäufigsten mit dem Ball-and-Socket Ankle assoziiert (77\%), die meist als talo-naviculare und/oder talo-kalkaneare Synostosen das untere Sprunggelenk blockieren. Strahldefekte bestehen in $52 \%$, wobei der Fuß meist vierstrahlig ist. Fusionen oder Agenesie von Ossa cuneiformia und von Metatarsalenbasen finden sich ebenfalls häufig. Auffällig ist, dass in den meisten Fällen, wie auch beim hier vorgestellten Patienten, das distale Fibula-Ende höher steht als normal und so eine Eversionsbewegung im oberen Sprunggelenk ermöglicht.

Noch immer wird kontrovers diskutiert, ob das Ball-and-Socket Ankle angeboren oder biomechanisch als Folge der subtalaren Fusion entstanden ist. In den Fällen, bei denen diese Fusion nicht vorhanden ist, bestehen häufig Anomalien im unteren Sprunggelenk, die den Bewegungsumfang einschränken, was für die biomechanische Genese spricht. Umgekehrt ist aber nur ein einziger Fall beschrieben, bei dem eine postnatale knöcherne Fusion im Subtalargelenk zu einem Ball-and-Socket Ankle führte, ohne dass eine weitere der typischen Begleitfehlbildungen vorhanden wäre (Takakura et al., J Bone Joint Surg 1986:
6813: 834 - 837). Bei allen übrigen angeführten Fällen bestehen zudem noch eine Beinlängendifferenz oder Oligodaktylie. Eine alleinige angeborene subtalare Fusion führt dagegen nicht notwendig zur Ausbildung eines talokruralen Kugelgelenks. Zumindest die Entwicklung des Ball-and-Socket Ankle scheint daher determiniert zu sein und den zentralen Bestandteil eines Symptomkomplexes darzustellen. Auch das praktisch mathematisch exakte Verhältnis von $\mathrm{m}: \mathrm{w}=2: 1$ spricht für einen genetischen Einfluss, es ist jedoch nur in einem einzigen Fall ein familiäres Auftreten beschrieben (Jacobs, Br J Radiol 1962; 35: $871-872$ ).

Es gibt mehrere Versuche, beim Balland-Socket Ankle ein klares Syndrom zu definieren (Bettin et al., J Pediatric Orthopaedics 1996; 16: 492 -496). Die Einzelsymptome korrelieren im Ausmaß aber nicht miteinander und sind in der Form zum Teil sehr unterschiedlich, so dass es schwierig ist, ein Syndrom abzugrenzen. Lediglich beim Metatarsus adductus varus findet sich meist eine beidseitige Ausbildung der Deformität ohne die typischen Begleitsymptome, was für ein eigenständiges Syndrom spricht.

Obwohl vom Sprunggelenk primär nur selten Probleme ausgehen und die Beinlängendifferenz offensichtlich und wesentlich bedeutender ist, ist die Kenntnis des Ball-and-Socket Ankle dennoch wichtig: Nach der Korrektur der Beinlängendifferenz können die anderen, assoziierten Symptome in den Vordergrund treten, so dass, wie beim hier vorgestellten Patienten, die Instabilität im Sprunggelenk doch klinische Bedeutung erlangen kann.

M. F. Maitz, M. Kellner, D. Hahn, Würzburg 